\title{
Introducción a la investigación contable en Brasil ${ }^{1}$
}

\author{
Hugo A. Macías ${ }^{2}$
}

Recibido: 14 de marzo de 2018

Aprobado: 12 de junio de 2018

\section{Clasificación JEL: M49}

\section{Resumen}

El objetivo del presente artículo fue presentar la estructura actual de la investigación contable en Brasil, con énfasis en tres eventos indexados, las 35 revistas contables y el papel de las maestrías y los doctorados. Para ello se llevó a cabo un análisis de contenido, a partir de una revisión sistemática de literatura publicada en las principales revistas contables brasileñas entre 2008 y 2013. Entre los hallazgos encontrados se destacaron las características de la contabilidad como campo científico en Brasil, características de los

1 Este trabajo fue presentado en el IV Congreso Nacional de Profesores de Contaduría Pública. Las memorias del evento se encuentran en el siguiente enlace: https://conveniocpc.org/images/Memorias_iv_encuentro/Mesa_Teoria_Contable/ Introducci\%C3\%B3n_a_la_investigaci\%C3\%B3n_contable_en_Brasil.pdf

Citar como: Macías. H. A. (2019). Introducción a la investigación contable en Brasil. Revista Activos, 16(30), 155-185. DOI: https://doi.org/10.15332/ 25005278.5064

2 Economista, M. Sc. en Economía, Ph. D. (c) en Administración, becario Colciencias para adelantar estudios de doctorado en Administración en la Universidad Eafit. Profesor asociado de la Universidad de Medellín en los programas de Contaduría Pública y Maestría en Tributación y Política Fiscal, excoordinador de la Sala de Ciencias Económicas y Administrativas de Conaces, Ministerio de Educación Nacional. Correos electrónicos: hmacias@udem.edu.co - hmaciasc@ eafit.edu.co. 
autores, evolución de las líneas de investigación y evolución de las revistas. La estructura reciente de la investigación contable en Brasil, auspiciada por el Ministerio de Educación más las exigencias de la Capes, hace que se convierta en un modelo maduro de investigación contable en Latinoamérica.

Palabras clave: investigación contable, Brasil, temas contables.

\title{
Introduction to accounting research in Brazil
}

\begin{abstract}
The objective of this paper is to present the current structure of accounting research in Brazil, with emphasis on three indexed events, the 35 accounting journals and the role of masters and doctorates. For this purpose, a content analysis was carried out, based on a systematic review of the literature published in the main Brazilian accounting journals between 2008 and 2013. Among the findings, the characteristics of accounting as a scientific field in Brazil, authors, evolution of the lines of research and evolution of the journals are highlighted. The recent structure of accounting research in Brazil, sponsored by the Ministry of Education and the requirements of Capes, makes it a mature model of accounting research in Latin America.
\end{abstract}

Keywords: Accounting research, Brazil, accounting issues. 


\section{Introducción}

"La diversidad es una fuente de generación de nuevas ideas". (Paula, Silva, Vieira y Coutinho, 2012, p. 47)

Ya es un lugar común que la investigación contable de corriente principal y corte positivista, se ha desarrollado especialmente en Estados Unidos y que las corrientes alternativas tienen su epicentro en el Reino Unido, con aportes importantes desde Australia, Canadá y Nueva Zelanda. La corriente principal se ocupa especialmente del área financiera, usa métodos cuantitativos y se basa en la economía, mientras que las corrientes crítica e interpretativa se ocupan de áreas diversas, usan especialmente métodos cualitativos y se basan en disciplinas como la sociología, la psicología y la antropología. Al parecer, en otras regiones del mundo se están consolidando procesos que están haciendo emerger una estructura policéntrica de la investigación contable mundial, como el caso de Brasil que se viene consolidando en la última década como un nuevo eje de ese policentrismo.

Ese proceso reciente de investigación contable en Brasil incluye autoevaluación colectiva permanente, discusiones públicas en congresos y publicaciones en revistas indexadas. El área de educación e investigación contable cuenta con un evento especializado y una revista (ambos indexados), donde se hace seguimiento a congresos, revistas, maestrías y doctorados, mediante revisiones sistemáticas de literatura que abordan aspectos como: 1) características del campo; 2) características de los autores;3) características de las redes; 4) evolución de las revistas; 5) evolución de cada área en congresos y revistas; 6) evolución de las maestrías y, 7) evolución de los doctorados.

El objetivo de esta ponencia es presentar un perfil de la investigación contable en Brasil, que ayude a los académicos hispanoparlantes a comprender la dinámica de ese nuevo eje de policentrismo y que sirva a estos mismos académicos como inventario de publicaciones para profundizar en sus propias áreas de interés. El método utilizado es la revisión sistemática de literatura aplicada a artículos sobre investigación contable publicados en revistas categoría A2, B1 y B2 (sistema Qualis-Capes), durante el período 2008-2013; es 
decir, se trata de una revisión de revisiones. Este tipo de trabajos no pretende agotar ninguno de los temas a los que hace referencia y sacrifica profundidad para poder dar una mirada amplia a la investigación en ese país. La ponencia solo tiene pretensiones de nivel introductorio, es una exploración preliminar.

A continuación, se presentan los aspectos metodológicos de la revisión; en segundo lugar, se muestra la revisión de ponencias presentadas en congresos; luego se expone la revisión de la literatura publicada en revistas y por último, se dan las conclusiones.

\section{Método utilizado para la revisión sistemática de literatura}

Para alcanzar el objetivo de presentar un perfil de la investigación contable en Brasil, en este trabajo se llevó a cabo una revisión sistemática de literatura, con base en la propuesta metodológica de Tranfield, Denyer y Smart (2003). Esta propuesta es utilizada como referencia metodológica en la revista International Journal of Management Reviews, que se dedica exclusivamente a revisiones de literatura y es publicada por la Academia Británica de Administración. El carácter "sistemático" de la revisión de literatura pretende disminuir la subjetividad de los investigadores que hacen la revisión, tanto en la elección de los documentos como en su análisis, y pretende ser un método replicable.

La revisión sistemática de literatura consiste en plantear unos objetivos y propósitos claros, definir métodos preplaneados, usar criterios explícitos y reproducibles en la selección de artículos para su revisión, buscar de manera exhaustiva todos los artículos potencialmente relevantes, evaluar la calidad de la investigación y la solidez de los resultados, así como presentar los resultados de manera equilibrada, imparcial y comprensible (Tranfield, Denyer y Smart, 2003).

Una vez definido el objetivo de la revisión, se procedió a identificar las 35 revistas contables que se encuentran indexadas y su categoría (Anexo 1). 
Luego se ingresó a la página web de las revistas categoría A2, B1 y B2 (las categorías más altas), donde se seleccionaron 60 artículos que analizan la investigación, bien sea en congresos, revistas, aspectos teóricos, aspectos metodológicos y características de los programas de maestría y doctorado (Tabla 1). Para el análisis de los artículos se conformó una carpeta con los 60 archivos en formato PDF y se definieron las categorías de análisis y de relación entre los textos, con apoyo en el software ATLAS.ti. Para la presentación de los resultados se analizaron por separado las dinámicas de los congresos y revistas.

Tabla 1. Artículos seleccionados para desarrollar la revisión sistemática de literatura

\begin{tabular}{|c|c|c|c|c|c|}
\hline 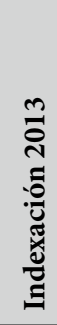 & Revista & 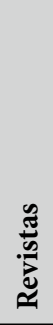 & 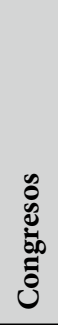 & 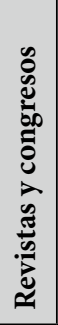 & 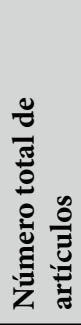 \\
\hline $\mathrm{A} 2$ & Revista Contabilidade \& Finanças & 1 & 1 & 0 & 2 \\
\hline \multirow{4}{*}{ B1 } & Contabilidade Vista \& Revista & 1 & 2 & 1 & 4 \\
\hline & Revista Contemporânea de Contabilidade & 5 & 3 & 0 & 8 \\
\hline & Revista de Contabilidade e Organizações & 2 & 3 & 0 & 5 \\
\hline & Revista Universo Contábil & 4 & 1 & 1 & 6 \\
\hline \multirow{5}{*}{ B2 } & Contabilidade, Gestão e Governança & 5 & 3 & 1 & 9 \\
\hline & Enfoque: Reflexão Contábil & 4 & 4 & 1 & 9 \\
\hline & Revista Ambiente Contábil & 2 & 0 & 2 & 4 \\
\hline & Revista de Administração, Contabilidade e Economia & 3 & 2 & 0 & 5 \\
\hline & Revista de Educação e Pesquisa em Contabilidade & 2 & 5 & 1 & 8 \\
\hline \multicolumn{2}{|c|}{ Total } & 29 & 24 & 7 & 60 \\
\hline
\end{tabular}

Fuente: elaboración propia. 


\section{Revisiones de las ponencias presentadas en eventos}

Uno de los mecanismos centrales que se ha utilizado en Brasil para construir conocimiento contable de manera colectiva, es evitar la proliferación de eventos desarticulados por todo el territorio nacional y concentrar los esfuerzos de posicionamiento y cualificación en un número pequeño de ellos. En las revisiones de literatura publicadas en revistas entre 2008 y 2013, se destacan cuatro eventos: 1) Congreso Brasilero de Costos (CBC); 2) encuentro de la Asociación Nacional de Posgrados e Investigación en Administración (Enanpad); 3) Congreso de la Universidad de Sao Paulo de Controladoria y Contabilidad (Congreso USP), y, 4) encuentro de la Asociación Nacional de Programas de Posgrado en Ciencias Contables (Anpcont). Todos ellos se realizan con periodicidad anual y están escalafonados en E1, la categoría de la Capes más alta para eventos.

\section{Características de los eventos}

El evento que ha acumulado una tradición más extensa es el Congreso Brasilero de Costos; este fue creado en 1994 con el nombre de gestión estratégica de costos, siguiendo la tendencia mundial de rediscusión y revalorización de los sistemas de costeo, inaugurada por un libro de contabilidad gerencial publicado por Johnson y Kaplan en 1993; el congreso es promovido por la Associação Brasileira de Custos, con dieciséis áreas temáticas. Fue creado con la expectativa de generar interacción de profesores y de estudiantes con empresarios, consultores, contadores, administradores y demás profesionales vinculados con la gestión estratégica de los costos; las ediciones anuales ininterrumpidas y sus avances cuantitativos y cualitativos le hicieron merecedor de la categoría A1 de Qualis-Capes en el año 2010 (Diehl y Souza, 2008; Souza y Rasia, 2011) y E1 en la actualidad.

Si bien el Enanpad es un encuentro de administración, que existía antes de 1998, es a partir de este año que la contabilidad empieza a ser considerada como área autónoma y fue el primer evento clasificado por la Capes como de nivel superior, que incluye trabajos contables, cuya participación 
se incrementó hasta el $12 \%$ en la primera década y se estabilizó allí (Araújo y Castro Silva, 2010; Silva, Wanderley y Santos, 2010). El Congreso USP se inició en el año 2001 en formato de seminario, donde se discutieron los trabajos de grado, disertaciones y tesis de ciencias contables producidos en la USP; de esta manera, la figura de Congreso USP de Controladoria y Contabilidad comenzó de manera abierta a partir de 2004, dirigido específicamente a la discusión de trabajos producidos en los posgrados (Cruz, Espejo, Costa y Almeida, 2011). Al interior del Congreso USP y otro similar de la Universidad Federal de Santa Catarina (UFSC), se desarrolla un congreso de "iniciación científica" en el que participan académicos con menor trayectoria como autores (Ensslin y Silva, 2008).

Tabla 2. Principales eventos de investigación contable en Brasil

\begin{tabular}{l|c|c|c|c}
\hline Congreso & $\begin{array}{c}\text { Nombre } \\
\text { común }\end{array}$ & Periodicidad & $\begin{array}{c}\text { Año de } \\
\text { inicio }\end{array}$ & $\begin{array}{c}\text { Estrato } \\
\text { Qualis 2013 }\end{array}$ \\
\hline Congreso Brasilero de Costos & CBC & Anual & 1994 & A \\
\hline $\begin{array}{l}\text { Encuentro de la Asociación } \\
\text { Nacional de Posgrados } \\
\text { e Investigación en } \\
\text { Administración }\end{array}$ & Enanpad & Anual & 1996 & A \\
\hline $\begin{array}{l}\text { Congreso Universidad de } \\
\text { Sao Paulo de Controladoria y } \\
\text { Contabilidad }\end{array}$ & $\begin{array}{l}\text { Congreso } \\
\text { USP }\end{array}$ & Anual & 2001 & A \\
\hline $\begin{array}{l}\text { Asociación Nacional de } \\
\text { Programas de Posgrado en } \\
\text { Ciencias Contables }\end{array}$ & Anpcont & Anual & 2007 & A \\
\hline
\end{tabular}

Fuente: elaboración propia

Por su parte, el congreso de Anpcont es un evento de importante significado académico y científico, que permite la interacción entre investigadores, profesores y estudiantes; también es un medio de divulgación técnico-científica en ciencias contables y busca establecer vínculos entre los estudios académicos y su aplicación a las organizaciones. Por ser un evento nacional, desde 2007 se ha realizado en diferentes ciudades brasileras, con lo cual se facilita la participación de un número amplio de integrantes de 
la comunidad académica; en las versiones recientes el evento ha adquirido carácter internacional (Matos, Niyama, Araujo Neto y Marques, 2012; Silva, Wanderley y Santos, 2010; Souza, Machado y Bianchi, 2011c).

\section{Fuentes utilizadas y método de investigación}

En Anpcont las principales referencias bibliográficas son artículos de revistas internacionales y el número de referencias ha crecido a lo largo del tiempo (Matos, Niyama, Araujo Neto y Marques, 2012); las ponencias presentadas en el mismo congreso son más citadas en contabilidad gerencial que en mercado de capitales, dado que la primera es un área más abierta y plural, mientras la segunda atiende más a criterios internacionales ${ }^{3}$ (Paula, Silva, Vieira y Coutinho, 2012). Por su parte, en el Congreso USP y en Enanpad, hay concentración de fuentes en libros de administración y contabilidad, que no se consideran una fuente actualizada para la producción de artículos académicos y para la solución de problemas actuales; el origen temático de los textos a los que se acude es considerado una fuente de "hermetismo contable"; la sociología, la psicología y la antropología son abordadas de manera casi exclusiva en el área de enseñanza e investigación contable (Cabral, Siqueira y Siqueira-Batista, 2011). En los eventos de "iniciación científica" de la USP y la UFSC, los libros son las obras más referenciadas y sobresalen las referencias nacionales (Ensslin y Silva, 2008). Todo indica que las fuentes utilizadas han avanzado más en la Anpcont que en los demás eventos.

Con respecto al método, las ponencias presentadas en el Congreso Anpcont son en su mayoría de investigación positiva, predominan los métodos empíricos, lo cual se ha acentuado a lo largo de los años (Paula, Silva, Vieira y Coutinho, 2012); algo similar ocurre en los eventos de "iniciación científica" de la USP y la UFSC, donde prevalecen los estudios empírico-teóricos (Ensslin y Silva, 2008). En el Congreso USP y en Enanpad, la interdisciplinariedad está restringida a economía, administración y

3 Un estudio que analiza los artículos sobre mercado de capitales publicados en revistas, llegó a la misma conclusión: las redes entre autores son dispersas y de lazos débiles (Nascimento, Pereira y Toledo Filho, 2010). 
educación (Cabral, Siqueira y Siqueira-Batista, 2011). El análisis de los trabajos presentados reveló de forma inequívoca que a lo largo de la primera década de publicación de artículos contables en los Enanpad, predominó la investigación positiva y desde el principio los trabajos normativos tendieron a desaparecer (Mendonça Neto, Riccio y Sakata, 2009)4. Las ponencias presentadas en versiones recientes del Congreso USP tienen inconsistencias en la formulación del problema en un $61 \%$, especialmente porque se trata de problemas implícitos, que se pueden responder de manera simple como "si" o "no"; en el $16 \%$ de los trabajos no se menciona el problema en la introducción de la ponencia (Cunha, Magro y Dias, 2012). Metodológicamente se resalta entonces la concentración reciente en investigación positiva, el bajo nivel de interdisciplinariedad y las autocríticas con respecto a una posición más clara de cada trabajo frente a la literatura que se ha publicado previamente.

\section{Características de los autores y las instituciones}

Uno de los trabajos clasificó a los ponentes de los cuatro congresos como entrantes, retirados, one-timers y continuadores, según su número de publicaciones en un período dado. Se consideran entrantes aquellos autores que han publicado varios artículos en los últimos años del período; retirados son aquellos que publicaban pero que no lo han hecho en los últimos dos años; one-timers son los que publicaron solo una vez en el período y continuadores son los autores que publican de manera periódica. Los continuadores son centrales en el desarrollo de la disciplina, porque tienen la mayor producción en cantidad e intermedian y relacionan las otras categorías de autores; es decir, agencian las informaciones de diferentes investigadores y además son los principales responsables de las publicaciones internacionales. En los cuatro congresos donde se discuten los resultados de investigación contable, indexados en estrato A por Qualis, la categoría con menor número

$4 \quad$ El artículo de Mendonça Neto, Riccio y Sakata (2009) está publicado en una revista de administración, pero fue adicionado aquí porque analiza la primera década de publicación de artículos contables en los Enanpad. 
de autores es la de continuadores y la de mayor número de autores es la de on-timers $^{5}$ (Walter, Bach, Souza y Frega, 2013).

Si bien los autores más prolíficos están vinculados a pocas instituciones, especialmente a la USP (Souza, Rover, Gallon y Ensslin, 2008), quienes presentan mayor número de ponencias en los distintos eventos son los que tienen mayor asociatividad (Freitas, Pacheco, Karolkievicz y Sillas, 2012b); es decir, la producción está concentrada en algunos estados, universidades y autores (Matos, Niyama, Araujo Neto y Marques, 2012), pero los autores con mayor productividad son los que articulan, los que crean vínculos, los que construyen centralidad en las redes. Esta interacción es importante, dado que en el Congreso USP y en el Congreso de Anpcont, la gran mayoría de los autores ha presentado una sola ponencia (Barbosa y Barros, 2010; Cruz, Espejo, Costa y Almeida, 2011) y son los continuadores los que retoman esas contribuciones para avanzar en la construcción colectiva de conocimiento. También se ha identificado un número alto de investigadores que tiene metas comunes y que vislumbran la internacionalización como una necesidad apremiante (Cruz, Espejo, Costa y Almeida, 2011).

Los autores de las ponencias presentadas en el Congreso USP y en el Congreso Anpcont cuentan con formación de posgrado: alrededor del 40 $\%$ tienen título de doctor y un $33 \%$ adicional tienen formación de maestría (Barbosa y Barros, 2010; Matos, Niyama, Araujo Neto y Marques, 2012). En esos mismos eventos lo más habitual es que haya dos autores por ponencia (Barbosa, Quintana y Machado, 2011), mientras que en los eventos de "iniciación científica" de la USP y la UFSC hay un número alto de autores por ponencia (Ensslin y Silva, 2008). En varias clasificaciones de autores construidas por los estudios que analizan los eventos, aparece la profesora

5 Las categorías de entrantes, retirantes, one-timers y continuantes, fueron adaptadas del trabajo de Guarido Filho, Machado-da-Silva y Goncalves, presentado como ponencia en el Enanpad de 2009 (Walter, Bach, Souza y Frega, 2013, p. 203). Las mismas categorías fueron utilizadas en el trabajo de Meli y Oliveira Neto (2011), que se presentará más adelante en este trabajo; en ese caso las categorías fueron adaptadas de la tesis doctoral de Guarido Filho, terminada en 2008 (Meli y Oliveira Neto, 2011, p. 159). 
Ilse Maria Beuren, liderando los autores más prolíficos y está acompañada en estos reportes de los profesores Adriano Leal Bruni, Auster Moreira Nascimento, Edgar Bruno Cornachine Jr., Edison Paulo, Fernando Dal-Ri Murcia, Gilberto de Andrade Martins, Luiz João Corrar, Maisa de Souza Ribeiro, Marcelo Alvaro da Silva Macedo y Maria Thereza Pompa Antunes ${ }^{6}$ (Barbosa et al., 2011; Freitas et al., 2012b; Luca et al., 2011; Souza et al., 2008). Estos autores que aparecen en los primeros lugares de los escalafones son los continuadores más destacados en los tres principales eventos.

Con respecto a las instituciones, en el trabajo de Souza et al. (2008) se hizo una clasificación sugestiva, a partir de las ponencias presentadas en los Congresos USP, Enanpad, Anpcont y el encuentro de enseñanza e investigación en administración y contabilidad (EnEPQ) ${ }^{7}$. En el estudio citado, los autores identificaron tres grupos de instituciones que presentan similitudes en la producción científica y en el número de investigadores: instituciones de centro, intermedias y de periferia. Por productividad científica, las instituciones del centro son USP, UnB, Fucape y UFSC; la zona intermedia está compuesta por 13 instituciones de educación superior y la periferia por 160. Esta productividad está vinculada directamente a la presencia de programas de maestría y doctorado en ciencias contables, que son los espacios de formación de investigadores ${ }^{8}$.

6 Algunos trabajos han analizado la participación femenina en los tres principales eventos contables en Brasil y han encontrado que es significativamente pequeña con relación al género masculino, aunque está aumentando (Barbosa y Barros, 2010; Luca et al., 2011).

7 No se tuvo en cuenta el Congreso Brasilero de Costos, porque en 2006 disminuyó su categoría a B.

8 En las IES de periferia se encontró poca tradición en investigación, aún en aquellas que cuentan con programas de maestría. Al parecer, los nuevos relacionamientos se dan a partir de los ya existentes y las IES de periferia tienen mayor dificultad para relacionarse con IES centrales (Souza et al., 2008, p. 33). 
Tabla 3. Revisiones sistemáticas de ponencias presentadas en congresos

\begin{tabular}{l|l}
\hline \multicolumn{1}{c|}{ Categoría } & \multicolumn{1}{c}{ Descripción } \\
\hline $\begin{array}{l}\text { Características } \\
\text { del campo }\end{array}$ & $\begin{array}{l}\text { Características de las ponencias (Matos et al., 2012); continuidad de } \\
\text { al., 2012b); definición del problema de investigación en ponencias } \\
\text { (Cunha et al., 2012); "diálogo científico" (Paula et al., 2012); diálogo } \\
\text { con las ciencias humanas (Cabral et al., 2011); perfil de ponencias de } \\
\text { iniciación científica (Ensslin y Silva, 2008) y redes en Congreso USP } \\
\text { (Cruz et al., 2011). }\end{array}$ \\
\hline $\begin{array}{l}\text { Características } \\
\text { de los autores }\end{array}$ & $\begin{array}{l}\text { Participación femenina en las ponencias (Luca et al., 2011); perfil de } \\
\text { los autores (Barbosa y Barros, 2010) y productividad de los investi- } \\
\text { gadores (Souza et al., 2008). }\end{array}$ \\
\hline $\begin{array}{l}\text { Evolución de } \\
\text { un tema en } \\
\text { uno o varios } \\
\text { congresos }\end{array}$ & $\begin{array}{l}\text { Alineamiento estratégico (Silva y Lunkes, 2011); contabilidad finan- } \\
\text { ciera (Silva et al., 2010); contabilidad gerencial (Araújo y Castro Silva, } \\
\text { 2010); controladoria (Beuren et al., 2012); controladoria y contabili- } \\
\text { dad gerencial (Ribeiro, 2013c); coordinación y control (Demuner } \text { et } \\
\text { al., 2008); costos ABC (Diehl y Souza, 2008); costos en administra- } \\
\text { ción pública (Carneiro, et al., 2012); costos en agronegocios (Souza } \\
\text { y Rasia, 2011); finanzas (Gubiani y Lavarda, 2011); gobernanza } \\
\text { (Duarte et al., 2012), gobierno corporativo (Campos et al., 2011) y } \\
\text { organizaciones sin ánimo de lucro (Olak et al., 2008). }\end{array}$ \\
\hline
\end{tabular}

Fuente: elaboración propia.

Entre las instituciones que participan más activamente en investigación, se da cierta especialización temática: USP, UnB, Fucape y UFPR concentran sus publicaciones en contabilidad para usuarios externos; UFSC y UFPE focalizan sus trabajos en educación e investigación contable, y en Mackenzie y UFMG predominan las investigaciones sobre mercados financieros, de crédito y de capitales. En general, las áreas de contabilidad gerencial y contabilidad para usuarios externos son predominantes en las IES más prolíficas, mientras que educación e investigación en contabilidad es el área menos explorada, a pesar de tener un encuentro especializado (EnEPQ) (Souza, Rover, Gallon y Ensslin, 2008). 


\section{Temas abordados en los eventos}

En el período 2008-2013, las 11 principales revistas contables de Brasil publicaron 13 artículos que analizan la evolución de un tema en uno o varios congresos. Entre esos trabajos, nueve estudiaron las ponencias publicadas en el Congreso USP, nueve examinaron las ponencias publicadas en los Enanpad, cinco analizaron las ponencias de Anpcont y cuatro trabajos escudriñaron ponencias presentadas en el Congreso Brasilero de Costos; es decir, los dos primeros son considerados por los investigadores como los principales espacios donde se discute cada tema?

Los cuatro trabajos que analizan simultáneamente los tres principales congresos (Congreso USP, Enanpad y Anpcont) coinciden en varios aspectos y se diferencian claramente en otros. En términos generales las ponencias de los diferentes temas coinciden: 1) en que prevalece el título de doctorado como formación de los autores; 2 ) el número de autores por artículo más común es de dos o tres; 3 ) la mayoría de los trabajos son aplicaciones a organizaciones específicas y, 4) en las fuentes prevalecen las referencias internacionales, con tendencia a aumentar. Sin embargo, las diferentes áreas temáticas tienen abordajes metodológicos distintos y son más estudiadas en algunas universidades. Por ejemplo, en alineamiento estratégico predominan los estudios exploratorios, prácticos y cualitativos (Silva y Lunkes, 2011), mientras que en contabilidad financiera más de la mitad de las ponencias utilizan abordaje estadístico, con 11 tipos de métodos estadísticos que están en aumento (Silva, Wanderley y Santos, 2010); por su parte, en finanzas corporativas ${ }^{10}$ prima lo descriptivo (Gubiani y Lavarda, 2011). Temáticamente también hay concentración en algunas instituciones: la USP

9 Cuatro trabajos analizan un tema en tres congresos: Enanpad, Anpcont y Congreso USP; dos trabajos analizan exclusivamente las ponencias de Enanpad; otros dos analizan exclusivamente las ponencias del Congreso USP y otros dos el Congreso Brasilero de Costos; los otros tres trabajos analizan los cuatro congresos simultáneamente, tres de ellos y uno de ellos.

10 El trabajo de Gubiani y Lavarda (2011) hace referencia a finanzas comportamentales y teoría de prospecto; aborda las finanzas desde la teoría del comportamiento orientadas a la toma de decisiones. 
aparece como la institución con mayor participación en gobierno corporativo, finanzas corporativas y contabilidad financiera, Fucape se destaca también en contabilidad financiera, pero en alineamiento estratégico las principales instituciones son UFRGS, UFRJ y FGV (Silva y Lunkes, 2011).

\section{Revisiones de la literatura publicada en revistas}

Entre las revisiones de literatura publicadas en el período 2008-2013, se encontraron artículos sobre la trayectoria de algunas revistas y otros dedicados a comparar dos revistas. El primer grupo de trabajos analiza la trayectoria de Contabilidade, Gestão e Governança (CGG); Revista Contemporânea de Contabilidade (RCC); Revista Contabilidade \& Finanças (RCF) y Revista de Educação e Pesquisa em Contabilidade (REPeC). El segundo grupo compara las revistas Contabilidade Vista \& Revista (CV\&R) con Revista Universo Contábil (RUC); Revista Contabilidade \& Finanças (RCF) con Base, y Revista Contabilidade \& Finanças (RCF) con The Accounting Review (TAR).

\section{Características de las revistas}

Las revistas CGG, CV\&R, RCC, REPeC y RUC, están indexadas en categoría B1 o B2 ${ }^{11}$, cuatro de ellas fueron creadas en el período 2004-2007 y en 2013 ya publicaban tres o cuatro números por año $0^{12}$; es decir, se trata de revistas muy jóvenes, que han logrado incrementar tempranamente su periodicidad y que han ascendido rápidamente en la escala nacional de indexación. Por su parte, RCF es la revista contable con más tradición en Brasil, creada en 1989 en la Universidad de Sao Paulo, es la única que ha llegado a la categoría

11 La escala de indexación de revistas en Brasil es A1, A2, B1, B2, B3, B4, B5 y C; A1 es la categoría más alta y C es la categoría más baja (Qualis-Capes, 2013). El nivel de indexación de las 34 revistas contables está en el Anexo 01 de este trabajo.

12 La revista CV\&R se creó desde 1989, cuando también fue creada RCF. Para 2013 CGG y RCC publicaban tres números al año y CV\&R, REPeC y RUC publicaban cuatro números anuales. La revista Base es de ciencias económicas, fue un referente central 10 años atrás para la investigación contable, pero ha perdido protagonismo por la cualificación formal de las revistas especializadas en contabilidad. 
A2 y siempre ha estado indexada en categoría superior a las demás ${ }^{13}$. TAR es la revista de la American Accounting Association que ocupa el primer lugar en todos los escalafones internacionales de revistas contables y se ha convertido en un referente explícito para RCF.

Desde el punto de vista temático, en RCF predominan los artículos de contabilidad financiera, seguidos de enseñanza de la contabilidad, teoría contable y sistemas de información (Brunozi Júnior et al., 2011). En RCC los temas más abordados han sido contabilidad de costos, enseñanza e investigación, contabilidad ambiental, gobierno corporativo y desempeño organizacional (Ribeiro, 2013a). Por su parte, en REPeC los temas más abordados son enseñanza e investigación, transparencia, contabilidad internacional, demostraciones contable-financieras, contabilidad gerencial, historia de la contabilidad, contabilidad gubernamental, mercado de capitales y controladoria (Ribeiro, 2013b); en CGG prevalecen los artículos sobre contabilidad gerencial (Perdigão, Niyama y Santana, 2010). Temáticamente es clara la diferencia entre RCF y las demás revistas, donde la primera está concentrada en contabilidad financiera, con enfoque cuantitativo positivista, de corriente principal en investigación contable; las demás revistas están orientadas a contabilidad gerencial, enseñanza e investigación contable y contabilidad ambiental, entre otros temas.

Metodológicamente, en RCF las publicaciones fueron de cuño bibliográfico y documental entre 1989 y 2002, pero entre 2003 y 2009 predominaron los estudios empíricos, con aporte de datos primarios y secundarios (Brunozi Júnior et al., 2011); la revista pasó de ser predominantemente normativa entre 1989 y 1997, al uso de "otros" abordajes entre 1998 y 2003, para acoger principalmente artículos positivistas entre 2004 y 2007 (Kroenke et al., 2011). Los artículos publicados en las demás revistas acuden a métodos diversos y un número mayor son de tipo cualitativo. Curiosamente, los investigadores son poco influenciados por las teorías y

13 La revista contó con la denominación de Caderno de Estudos en el período 1989-2000 y adquirió la denominación de Revista Contabilidade \& Finanças a partir de 2001. 
Tabla 3. Revisiones sistemáticas de literatura publicada en revistas

\begin{tabular}{|c|c|}
\hline Categoría & Descripción \\
\hline Evolución de una revista & $\begin{array}{l}\text { Contabilidade, Gestão e Governança (Esteves y Botelho, } \\
\text { 2013; Perdigão et al., 2010); Revista Contabilidade \& } \\
\text { Finanças (Brunozi Júnior et al., 2011; Kroenke et al., } \\
\text { 2011); Revista Contemporânea de Contabilidade (Ribeiro, } \\
\text { 2013a) y Revista de Educação e Pesquisa em Contabilidade } \\
\text { (Ribeiro, 2013b). }\end{array}$ \\
\hline $\begin{array}{l}\text { Comparación de dos } \\
\text { revistas }\end{array}$ & $\begin{array}{l}\text { Contabilidade Vista \& Revista vs. Revista Universo Contábil } \\
\text { (Lima et al., 2013); Revista Contabilidade \& Finanças vs. } \\
\text { Base (Batistella et al., 2008) y Revista Contabilidade \& } \\
\text { Finanças vs. The Accounting Review (Dantas et al., 2011). }\end{array}$ \\
\hline $\begin{array}{l}\text { Evolución de un tema en } \\
\text { una o varias revistas }\end{array}$ & $\begin{array}{l}\text { Ajustes contables (Andrade et al., 2011); contabilidad } \\
\text { ambiental (Freitas et al., 2012a; Rosa } \text { et al., 2012; Silva y } \\
\text { Pires, 2013); controladoria (Beuren y Silva, 2010); costos } \\
\text { (Machado et al., 2012); gobierno corporativo (Catapan y } \\
\text { Cherobim, 2010); flujos de caja (Barbosa et al., 2011) y } \\
\text { presupuesto (Suave et al., 2013). }\end{array}$ \\
\hline $\begin{array}{l}\text { Procesos de gestión de } \\
\text { las revistas }\end{array}$ & $\begin{array}{l}\text { Celeridad en la evaluación de artículos (Dias et al., 2011); } \\
\text { comparación entre revistas disponibles y necesidades } \\
\text { adicionales de espacios de publicación (Murcia et al., } \\
\text { 2013); proceso editorial (Souza et al., 2011b) y propuesta } \\
\text { de indexación interna de revistas extranjeras (Murcia y } \\
\text { Borba, 2008). }\end{array}$ \\
\hline Características del campo & $\begin{array}{l}\text { Cambio de paradigma (Mendonça Neto et al., 2008); } \\
\text { evolución general del campo (Avelar et al., 2012) y mapa } \\
\text { de redes de colaboración (Silva et al., 2012). }\end{array}$ \\
\hline $\begin{array}{l}\text { Características de los } \\
\text { autores }\end{array}$ & $\begin{array}{l}\text { Continuidad de los autores (Meli y Oliveira Neto, 2011) y } \\
\text { productividad de los docentes permanentes de } 18 \text { maes- } \\
\text { trías contables (Soares et al., 2013). }\end{array}$ \\
\hline
\end{tabular}

Fuente: elaboración propia.

métodos de los orientadores de maestría y doctorado, son más direccionados por los eventos académicos (Kroenke et al., 2011).

Con respecto a los autores, en las cuatro revistas (CGG, RCC, RCF y $\mathrm{REPeC}$ ) la mayoría son doctores o estudiantes de doctorado y muy pocos cuentan solo con título de pregrado. Esto ocurre porque el Ministerio de 
Educación exige que los profesores vinculados a los posgrados tengan publicaciones científicas relevantes y es en esos programas académicos donde se genera el conocimiento contable producido en Brasil, por medio de artículos escritos en coautoría. A partir de 2003 disminuyó la concentración en autores de la USP por la creación de programas de maestría y doctorado en otras instituciones (Brunozi Júnior, Emmendoerfer, Abrantes y Klein, 2011; Ribeiro, 2013a).

Entre los profesores más prolíficos y con alto grado de centralidad en las redes, se destacan Ilse Maria Beuren en CGG y RCC (Esteves y Botelho, 2013; Ribeiro, 2013a); Romualdo Douglas Colauto en RCC (Ribeiro, 2013a); Eliseu Martins en RCF (Brunozi Júnior et al., 2011; Kroenke et al., 2011), así como Aridelmo José Campanharo Teixeira y Gilberto de Andrade Martins en REPeC (Ribeiro, 2013b). En los primeros años de todas las revistas, a medida que va pasando el tiempo aumenta el número de coautores por artículo, disminuye la participación de autores vinculados con la institución editora de la revista y se va posicionando un número pequeño de autores en la centralidad de las redes.

Las comparaciones entre revistas, resaltan que tanto Base como CV\&R, RCF, RUC y TAR, son estables en el número de artículos publicados por edición, pero TAR publica más artículos cada año ya que tiene seis ediciones anuales, mientras las otras tienen tres o cuatro ediciones por año (Batistella et al., 2008; Dantas et al., 2011; Lima et al., 2013). Con respecto a los autores, las características son muy parecidas en las cinco revistas: un promedio de dos autores por artículo, autocitaciones en alrededor del $3 \%$ y reducción del número de coautores vinculados a la misma institución. El perfil de la producción científica es muy similar en CV\&R, RCF y RUC, mientras que la revista Base cada vez publica menos artículos contables ${ }^{14}$.

14 La revista Base es una publicación genérica de ciencias económicas que fue central en la producción académica contable 10 años atrás, pero ha perdido protagonismo por el avance formal de las revistas especializadas en contabilidad. 
La comparación entre RCF y TAR revela importantes retos de fondo y de forma para la primera, que intencionalmente está construyendo un perfil similar. En primer lugar, los patrones de producción científica (estructura de los artículos) en Brasil siguen siendo diferentes a los patrones internacionales de corriente principal, lo cual al parecer explica la poca inserción brasileña en revistas internacionales. En segundo lugar, las referencias bibliográficas en las que se apoyan los artículos publicados en TAR son mayores en valor absoluto, están más concentradas en revistas que en libros y tienen menor participación de tesis y disertaciones (Dantas et al., 2011). Tanto la estructura de los artículos como los autores que participan y las fuentes en las que se apoyan, revelan que persiste una distancia importante entre RCF y TAR.

La literatura contable de Brasil también aborda los procesos de gestión editorial de las revistas, tanto desde el tiempo que tarda en publicarse un artículo postulado y los criterios de cualificación académica de las revistas, hasta la categoría de indexación en la que deben estar las revistas extranjeras y la cantidad de revistas necesarias para tejer las relaciones académicas entre una comunidad contable del tamaño de la brasileña. En primer lugar, se encontró que en el período 2004-2009 aumentó el tiempo que tarda en publicarse un artículo, desde que fue postulado; esto ocurre por los procesos formales para mejorar el nivel de indexación, el aumento en el número de artículos postulados y el bajo número de evaluadores, entre otros (Dias, Barbosa Neto y Cunha, 2011). En segundo lugar, se propuso un conjunto de objetivos para una revista en particular, que pueden ser utilizados por otras para avanzar en sus procesos de cualificación académica, en la categoría formal de indexación y en su reconocimiento internacional; los 13 objetivos propuestos están distribuidos en tres perspectivas: sociedad, procesos internos y "aprendizaje y crecimiento" (Souza, Petri y Cardoso, 2011b).

Así mismo, en un trabajo se presentó una metodología para evaluar las revistas contables extranjeras y asignar la categoría en el proceso de homologación de la Capes (Murcia y Borba, 2008); los investigadores plantearon cuáles revistas extranjeras deben estar indexadas y en qué categoría, en lugar de esperar que lo definan funcionarios menos familiarizados con este campo 
científico en particular. Por su parte, un análisis riguroso sobre el número de programas de maestría y doctorado, reveló que el crecimiento de estos ha motivado el aumento en el número y cualificación de las revistas, pero al mismo tiempo mostró que los puntos disponibles en las revistas especializadas en contabilidad no son suficientes para responder a las exigencias de publicación por parte de la Capes (Murcia, Rosa y Borba, 2013). Ningún país de Latinoamérica tiene 34 revistas contables indexadas como Brasil, pero en este país la dinámica de esta comunidad hace que sean insuficientes estas 34 revistas, muchas de ellas con tres y cuatro ediciones anuales.

\section{Temas abordados en las revisiones}

Sobre contabilidad ambiental se analizaron tres artículos recientes que revisan la literatura publicada en revistas. En el primero de ellos se estudiaron las revistas de las instituciones que tienen doctorado en contabilidad y se encontró que existen pocas publicaciones sobre el tema y que en lo existente se destaca la aplicabilidad y evidenciación de factores ambientales (Freitas, Quaresma, Schmitt, Gonçalves y Quintana, 2012a). Sin embargo, en otro trabajo se revisó lo publicado durante los últimos 20 años en revistas de estrato Qualis A, B y C, y se llegó a la conclusión de que en los últimos años se han dado grandes pasos para evaluar y atender las necesidades sociales, económicas y financieras de la sociedad (Silva y Pires, 2013). En el mismo sentido, el tercer trabajo revisó 103 artículos publicados en el período 1991-2010 y concluyó que la contabilidad ambiental se consolidó en Brasil a partir de 2007, con unos énfasis específicos (Rosa, Voss, Ensslin y Ripoll Feliu, 2012).

Por su parte, en contabilidad de gestión se han publicado revisiones recientes de la literatura de costos, presupuesto y flujos de caja. Los 80 artículos sobre costos publicados en nueve revistas tratan cuatro temas en particular sin que uno de ellos se destaque más; las redes entre investigadores están en fase embrionaria y se ha avanzado bastante en la construcción de casos (Machado, Silva y Beuren, 2012); en el mismo sentido, una revisión sobre teoría de ajustes contables en las principales revistas, encontró que 
los artículos defienden la idea de que las informaciones contables basadas solamente en el costo histórico no revelan la situación real de los patrimonios de las empresas (Andrade, Segantini y Silva, 2011). Sobre presupuesto se analizaron 19 artículos publicados en revistas de estrato Qualis B1 y B2, y se encontró que no hay autores que predominen en el tema dentro de Brasil y que también se ha avanzado en la construcción de casos (Suave, Lunkes, Rosa y Soares, 2013). Con respecto a flujos de caja, se encontró un número pequeño de artículos publicados en los primeros 20 años de RCF, pero allí se identificó el autor seminal, los principales temas abordados y el origen de las referencias, entre otros (Barbosa, Quintana y Machado, 2011).

En la revisión de 26 artículos sobre gobierno corporativo publicados entre 2000 y 2010, se encontró predominio de trabajos empíricos, ausencia de trabajos normativos, la USP es la institución más prolífica y no se distinguen sectores económicos (Catapan y Cherobim, 2010). Sobre controladoria, a pesar de ser un concepto presente en la denominación de algunos programas académicos, se hallaron vacíos conceptuales y pocos avances procedimentales y organizacionales (Beuren y Silva, 2010).

Algunas revisiones destacaron la ausencia de marco conceptual en el subcampo estudiado (Rosa, Voss, Ensslin y Ripoll Feliu, 2012), o identificaron que solo se utilizan algunas categorías del marco identificado por los investigadores (Beuren y Silva, 2010). El método más utilizado en contabilidad gerencial es el estudio de caso, tanto en la literatura sobre presupuesto (Suave, Lunkes, Rosa y Soares, 2013), como en la de costos (Machado, Silva y Beuren, 2012); mientras que en la literatura sobre gobierno corporativo existe una tendencia a recolectar datos usando el software Economatica (Catapan y Cherobim, 2010).

\section{Características del campo científico y los autores}

Sobre el paradigma de investigación adoptado por la investigación contable en Brasil, hay dos trabajos muy interesantes que se publicaron en el período analizado; uno de ellos plantea que se presentó una mudanza de paradigma 
a mediados del siglo pasado y el otro indica que recientemente el paradigma de investigación brasileño volvió a cambiar. Por un lado, se define que a mediados del siglo pasado pasó a predominar el pensamiento contable norteamericano, en detrimento de la escuela italiana que había prevalecido hasta entonces. Ese cambio de paradigma se explicó de manera empírica y con fundamentos teóricos, mediante la aplicación de las etapas de la sociología de la traducción, 1) problematización; 2) persuasión; 3) alistamiento y, 4) movilización de aliados) a 777 artículos publicados entre 1912 y 1979. Allí se identificaron tanto los principales interesados en el cambio, como los métodos de persuasión y alistamiento utilizados en la consolidación del proceso, a través de la movilización de aliados (Mendonça Neto et al., 2008).

Por su parte, en otro trabajo se estudiaron las investigaciones empíricas en contabilidad publicadas en las principales revistas contables nacionales en el período 2000-2009. Allí se encontró que: 1) el número de artículos de contabilidad financiera es bastante superior a las demás áreas (contabilidad gerencial y enseñanza e investigación en contabilidad); 2) que el abordaje cuantitativo predomina y está en aumento y; 3) que para recolectar los datos predominan los métodos de investigación documental y bases de datos externas (Avelar et al., 2012). Es decir, al parecer desde 2006 la investigación contable está migrando de nuevo, desde la contabilidad de gestión hacia la contabilidad financiera, con aumento de métodos cuantitativos; cada vez la investigación contable en Brasil toma más características de la investigación contable de corriente principal en el mundo anglosajón. Este último proceso coincide con el incremento de las redes de colaboración entre académicos de distintas instituciones, especialmente profesores de maestrías y doctorados en ciencias contables (Silva et al., 2012).

Uno de los trabajos que analizó la producción académica de siete revistas contables fue más allá de la descripción de las redes, para analizar y explicar el perfil de colaboración científica (Meli y Oliveira Neto, 2011). Ese trabajo encontró que la red está fragmentada; que un componente grande está aislado: el $71 \%$ de los autores publicaron apenas una vez entre 2005 y 2009; solo $2.5 \%$ de los autores publican continuamente; el $48 \%$ de los trabajos tienen alguna relación entre sí, pero los demás están "pulverizados", 
no tienen ningún vínculo con el componente principal de la red; 138 de los autores estudiados están vinculados solo en parejas o están totalmente aislados. Cuando se retiran los autores que publican una sola vez y los que publican de manera aislada, la red deja de estar fragmentada (Meli y Oliveira Neto, 2011).

Para analizar la interacción entre los autores contables, Meli y Oliveira Neto (2011) retomaron las categorías de one-timers, entrantes, retirados y continuadores. Los primeros son aquellos que publican una sola vez en un período dado; los segundos son los que han publicado varias veces, pero al final del período considerado; los retirados han publicado varias veces, pero no en los últimos años del período, y los continuadores son los autores que publican periódicamente. En las siete revistas contables analizadas entre 2005 y 2009, participaron 939 autores, de los cuales 667 son one-timers y solo 23 son continuadores. Lo interesante es que los autores continuadores son los que tienen mayor centralidad de grado en la red, son los que atraen más colaboradores y tienen mayor prestigio; los continuadores son los autores que guían la investigación académica y vinculan el trabajo colectivo; usualmente tienen título de doctor y trabajan como profesores de maestrías y doctorados en diferentes ciudades.

La productividad de los docentes de 18 maestrías contables sigue concentrada en un pequeño número de instituciones, a pesar de la reciente creación de programas de maestría a lo largo del territorio nacional. Tanto la producción más alta por programa de maestría, como la productividad más alta por profesor y la publicación en revistas internacionales, se concentran en USP, FURB, UFSC y Fucape. En Brasil, la publicación científica es un factor importante en la evaluación de los programas de maestría y doctorado; esa productividad explica en gran medida la nota que la Capes le otorga a los programas, que van de 1 a 5 para las maestrías y de 1 a 7 para los doctorados; la nota mínima para mantenerse en funcionamiento es de 3 (Soares et al., 2013). En buena medida, el hecho de que la producción esté concentrada en las maestrías y los doctorados es resultado de las exigencias institucionales de la Capes. 


\section{Conclusiones}

El objetivo de este trabajo fue construir un perfil de la investigación contable en Brasil, a partir de un grupo de eventos académicos de alto nivel y de artículos publicados en revistas contables indexadas. El nivel de madurez al que han llegado los principales eventos académicos, así como el crecimiento reciente en el número de revistas contables y su ascenso en el nivel de indexación, han hecho posible que los avances en la investigación contable se conviertan en objeto de investigación para la línea de "educación e investigación en contabilidad”; esta línea cuenta con su propio evento y su propia revista, pero que a su vez se desarrolla en todos los eventos y la mayoría de las revistas indexadas.

Para construir el perfil de la investigación contable en Brasil, se llevó a cabo una revisión sistemática de literatura, siguiendo lineamientos internacionales aceptados en la academia de administración y contabilidad. Tanto en el caso de los eventos como de la evolución de las revistas, se eligieron artículos publicados en revistas indexadas, que analizaran los eventos o las revistas, es decir, se llevó a cabo una revisión de revisiones.

Con respecto a los eventos se encontró que hay cuatro congresos consolidados en los que se presentan los resultados de la investigación contable, todos indexados en categoría A y con periodicidad anual. Metodológicamente se ha dado una concentración reciente en investigación positiva y bajo nivel de interdisciplinariedad. En las fuentes utilizadas, se acude principalmente a libros de administración y contabilidad, que no se consideran una fuente actualizada para la producción de artículos académicos, pero en uno de los eventos se ha avanzado más en la cualificación de las fuentes. En la academia brasileña se hace la diferencia entre los autores que hacen contribuciones sistemáticas a la literatura y los que han tenido una participación ocasional. Además de la USP, que es la líder tradicional de la investigación contable en Brasil, se destacan en un nivel central las instituciones UnB, Fucape, UFSC, UFPR, UFMG y Mackenzie. 
Por su parte, la mayoría de las 35 revistas contables indexadas son jóvenes (fueron creadas hace menos de 10 años), pero ya publican tres o cuatro números anuales y han ascendido rápidamente en el nivel de indexación local. Cada una tiene su propio perfil, son diferenciables temática y metodológicamente, una ha tomado el liderazgo históricamente, hay evaluación de la trayectoria individual, comparación entre revistas y comparación formal con la revista que se ha convertido en el principal referente internacional en investigación contable. También hay análisis de la evolución de un tema en una o varias revistas y análisis formal de los procesos de gestión de las revistas contables, recomendaciones de cualificación, así como características del campo y características de los autores.

La conclusión general es que la investigación contable en Brasil se ha consolidado en la última década en su entorno nacional. Para llegar a ese nivel se ha beneficiado del ambiente académico nacional, centrado en universidades federales a las que el Ministerio de Educación hace un seguimiento exigente, riguroso y periódico, especialmente en lo relacionado con la presentación de ponencias y publicación de artículos por parte de los docentes, resultado de disertaciones de maestría y tesis doctorales. Así mismo, se ha construido una tradición académica contable, desde el doctorado de la USP iniciado en 1978, las 19 maestrías contables que están en funcionamiento, la consolidación de cuatro congresos y el avance acelerado de 35 revistas contables indexadas.

\section{Referencias}

Andrade, J. M., Segantini, G. T. y Silva, J. D. (2011). Análise dos perfis dos artigos com estrato qualis Capes sobre teoria dos ajustamentos contábeis do lucro. Enfoque: Reflexão Contábil, 30(3), 33-43.

Araújo, E. A. y Castro Silva, W. A. (2010). Pesquisa científica em contabilidade gerencial nos Enanpads de 2003 a 2008. Revista Universo Contábil, 6(3), 2944. DOI: https://doi.org/10.4270/ruc.2010320

Barbosa, D. S., Quintana, A. C. y Machado, D. G. (2011). Análise da produção científica sobre os fluxos de caixa e a demonstração dos fluxos de caixa: um 
estudo da Revista de Contabilidade e Finanças da Universidade de São Paulo, no período de 1989 a 2009. Enfoque: Reflexão Contábil, 30(2), 52-66. DOI: https://doi.org/10.4025/enfoque.v30i2.12452

Barbosa, G. C. y Barros, F. O. (2010). Perfil dos autores na produção científica em contabilidade: o caso do congresso USP de controladoria e contabilidade e do congresso Anpcont. Enfoque: Reflexão Contábil, 29(3), 22-33.

Beuren, I. M. y Silva, A. J. (2010). Abordagens da controladoria em artigos publicados em periódicos dos programas de pós-graduação em ciências contábeis recomendados pela Capes. Enfoque: Reflexão Contábil, 29(3), 9-21.

Brunozi Júnior, A. C., Emmendoerfer, M. L., Abrantes, L. A. y Klein, T. C. (2011). Revista Contabilidade \& Finanças - USP: uma análise do perfil da produção científica de 1989 a 2009. Revista Universo Contábil, 7(4), 39-59. DOI: https:// doi.org/10.4270/ruc. 2011430

Cabral, I., Siqueira, J. R. y Siqueira-Batista, R. (2011). O diálogo das ciências contábeis com as ciências humanas e demais ciências sociais: uma análise da produção acadêmica do Congresso USP de controladoria e contabilidade e Enanpad (2004-2007). Revista de Educação e Pesquisa em Contabilidade, 5(4), 100-125.

Capes. (10 de junio de 2010). Coordenação de Aperfeiçoamento de Pessoal de Nível Superior . Recuperado el 10 de diciembre de 2013, de Diretoria de Avaliação - DAV:http://www.capes.gov.br/images/stories/download/avaliacao/ ADMIN17jun10.pdf

Catapan, A. y Cherobim, A. P. (2010). Estado da arte da governança corporativa: estudo bibliométrico nos anos de 2000 a 2010. RACE - Revista de Administração, Contabilidade e Economia, 9(1-2), 207-230.

Cruz, A. P., Espejo, M. M., Costa, F. y Almeida, L. B. (2011). Perfil das redes de cooperação científica: congresso USP de controladoria e contabilidade - 2001 a 2009. Revista Contabilidade \& Finanças, 22(55), 64-87. DOI: https://doi. org/10.1590/S1519-70772011000100005

Cunha, P. R., Magro, C. B. y Dias, D. R. (2012). Análise do problema de pesquisa dos artigos científicos publicados no $11^{\circ}$ Congresso USP de Controladoria e Contabilidade. Revista de Contabilidade e Organizações, 6(15), 123-141. DOI: https://doi.org/10.11606/rco.v6i15.52660

Dias, W. O., Barbosa Neto, J. E. y Cunha, J. V. (2011). A comunicação do conhecimento científico: dados sobre a celeridade do processo de avaliação e de 
publicação de artigos científicos em periódicos da área de contabilidade. Revista Contemporânea de Contabilidade, 8(15), 41-62. DOI: https://doi. org/10.5007/2175-8069.2011v8n15p41

Diehl, C. A. y Souza, M. A. (2008). Publicações sobre o custeio baseado em atividades (ABC) em congressos brasileiros de custos no período de 1997 a 2006. Contabilidade Vista \& Revista, 19(4), 39-57.

Ensslin, S. R. y Silva, B. M. (2008). Investigação do perfil dos artigos publicados nos congressos de contabilidade da USP e da UFSC com ênfase na iniciação científica. Revista de Contabilidade e Organizações, 3(2), 113-131. DOI: https:// doi.org/10.11606/rco.v2i3.34716

Esteves, L. L. y Botelho, D. R. (2013). Autores centrais no desenvolvimento da rede colaborativa: análise da revista Contabilidade, Gestão e Governança de 1998 a 2012. Contabilidade, Gestão e Governança, 16(3), 41-57.

Freitas, D. P., Quaresma, J. C., Schmitt, S. R., Gonçalves, T. L. y Quintana, A. C. (2012a). Contabilidade ambiental: um estudo bibliométrico em revistas científicas brasileiras. Revista Ambiente Contábil, 4(1), 72-88.

Freitas, E. M., Pacheco, V., Karolkievicz, R. M. y Sillas, E. P. (2012b). Cooperação acadêmica: análise de publicações em eventos científicos sobre ensino e pesquisa em contabilidade. Revista de Educação e Pesquisa em Contabilidade, 6(4), 399-418. DOI: https://doi.org/10.17524/repec.v6i4.218

Gubiani, C. A. y Lavarda, C. E. (2011). Perfil da produção bibliográfica sobre finanças comportamentais e teoria do prospecto. RACE - Revista de Administração, Contabilidade e Economia, 10(2), 163-184.

Machado, D. G., Silva, T. P. y Beuren, I. M. (2012). Produção científica de custos: análise das publicações em periódicos nacionais de contabilidade sob a perspectiva das redes sociais e da bibliometria. Contabilidade, Gestão e Governança, 15(3), 3-16.

Matos, E. B., Niyama, J. K., Araujo Neto, L. M. y Marques, M. M. (2012). Congresso Anpcont: análise bibliométrica descritiva e avaliativa dos artigos publicados de 2007 a 2011. Enfoque: Reflexão Contábil, 31(3), 73-88. Doi: https://doi. org/10.4025/enfoque.v31i3.16946

Meli, D. B. y Oliveira Neto, J. D. (2011). O perfil da colaboração nos periódicos contábeis nacionais: muitos one-timers e poucos continuants. Revista 
Contemporânea de Contabilidade, 8(15), 151-176. DOI: https://doi. org/10.5007/2175-8069.2011v8n15p151

Mendonça Neto, O. R., Riccio, E. L. y Sakata, M. C. (2009). Dez anos de pesquisa contábil no Brasil: análise dos trabalhos apresentados nos Enanpads de 1996 a 2005. RAE - Revista de Administração de Empresas, 49(1), 62-73. DOI: https:// doi.org/10.1590/S0034-75902009000100008

Murcia, F. C., Rosa, C. A. y Borba, J. A. (2013). Produção científica em ciências contábeis: uma comparação entre a meta estabelecida pela Capes e a publicação de artigos por parte dos docentes de programas de pós-graduação. Contabilidade, Gestão e Governança, 16(1), 68-81.

Murcia, F. D. y Borba, J. A. (2008). Possibilidades de inserção da pesquisa contábil brasileira no cenário internacional: uma proposta de avaliação dos periódicos científicos de contabilidade e auditoria publicados em língua inglesa e disponibilizados no portal de periódicos da Capes. Revista Contabilidade \& Finanças, 19(46), 30-43. DOI: https://doi.org/10.1590/S1519-70772008000100004

Nascimento, S., Pereira, A. M. y Toledo Filho, J. R. (2010). Produção científica em periódicos de contabilidade relacionada ao mercado de capitais. Contabilidade, Gestão e Governança, 13(1), 32-43.

Paula, M. M., Silva, A. J., Vieira, J. y Coutinho, A. (2012). Diálogo científico nos congressos Anpcont: diversidade inovadora ou isomorfismo institucionalizado? Contabilidade, Gestão e Governança, 15(3), 35-51.

Perdigão, L. Z., Niyama, J. K. y Santana, C. M. (2010). Contabilidade, gestão e governança: análise de doze anos de publicação (1998 a 2009). Contabilidade, Gestão e Governança, 13(3), 3-16.

Qualis-Capes. (10 de diciembre de 2013). Web Qualis. Recuperado el 10 de diciembre de 2013, de Sistema Integrado Capes - Sicapes: http://qualis.capes.gov.br/ webqualis/principal.seam

Ribeiro, H. C. (2013a). Revista Contemporânea de Contabilidade: uma análise do perfil da produção acadêmica durante o período de 2004 a 2012. Revista Contemporânea de Contabilidade, 10(20), 3-28. Doi: https://doi. org/10.5007/2175-8069.2013v10n20p3

Ribeiro, H. C. (2013b). Características da produção veiculada na Revista de Educação e Pesquisa em Contabilidade no período de 2007 a 2012. Revista 
de Educação e Pesquisa em Contabilidade, 7(4), 424-443. DOI: https://doi. org/10.17524/repec.v7i4.973

Rosa, F. S., Voss, B. L., Ensslin, S. R. y Ripoll Feliu, V. (2012). Evidenciação ambiental: estudo comparativo das contribuições teórico-metodológicas de Brasil e Espanha. Revista Universo Contábil, 8(1), 123-140. DOI: https://doi. org/10.4270/ruc.2012108

Silva, A. C., Wanderley, C. A. y Santos, R. (2010). Utilização de ferramentas estatísticas em artigos sobre Contabilidade Financeira - um estudo quantitativo em três congressos realizados no país. Revista Contemporânea de Contabilidade, $7(14), 11-28$.

Silva, F. M. y Lunkes, R. J. (2011). Análise dos artigos científicos brasileiros sobre o alinhamento estratégico: um estudo das publicações nos principais eventos de contabilidade no período de 2004 a 2009. Enfoque: Reflexão Contábil, 30(1), 35-48. DOI: https://doi.org/10.4025/enfoque.v30i1.12077

Silva, P. Y. y Pires, J. S. (2013). Análise da produção científica brasileira em contabilidade voltada para o segmento de gestão social e ambiental. Revista Ambiente Contábil, 5(1), 200-223.

Souza, F. C., Rover, S., Gallon, A. V. y Ensslin, S. R. (2008). Análise das IES da área de ciências contábeis e de seus pesquisadores por meio de sua produção científica. Contabilidade Vista e Revista, 19(3), 15-38.

Souza, J. V., Petri, S. M. y Cardoso, M. S. (2011b). Uma contribuição à avaliação dos periódicos científicos por meio do Balanced Scorecard: ilustração da avaliação e operacionalização da gestão estratégica na RCC. Revista Contemporânea de Contabilidade, 8(15), 87-104. DOI: https://doi. org/10.5007/2175-8069.2011v8n15p87

Souza, M. A. y Rasia, K. A. (2011). Custos no agronegócio: um perfil dos artigos publicados nos anais do congresso brasileiro de custos no período de 1998 a 2008. Contabilidade, Gestão e Governança, 14(1), 69-81.

Souza, M. A., Machado, D. G. y Bianchi, M. (2011c). Um perfil dos programas brasileiros de pós-graduação stricto sensu em contabilidade. Revista de Educação e Pesquisa em Contabilidade, 5(2), 67-95. DOI: https://doi.org/10.17524/ repec.v5i2.344

Suave, R., Lunkes, R. J., Rosa, E. C. y Soares, S. V. (2013). Orçamento: análise das publicações nas revistas de contabilidade do Brasil. RACE - Revista de 
Administração, Contabilidade e Economia, 12(2), 641-676. DOI: https://doi. org/10.18593/race.v12i2.4832

Tranfield, D., Denyer, D. y Smart, P. (2003). Towards a methodology for developing evidence-informed management knowledge by means of systematic review. British Journal of Management, 14(3), 207-222. DOI: https://doi. org/10.1111/1467-8551.00375

Walter, S. A., Bach, T. M., Souza, M. J.y Frega, J. R. (2013). Permanência e inserção de atores na produção científica de 1994 a 2009 na área de contabilidade. Revista de Educação e Pesquisa em Contabilidade, 7(2), 199-214. DOI: https:// doi.org/10.17524/repec.v7i2.633 


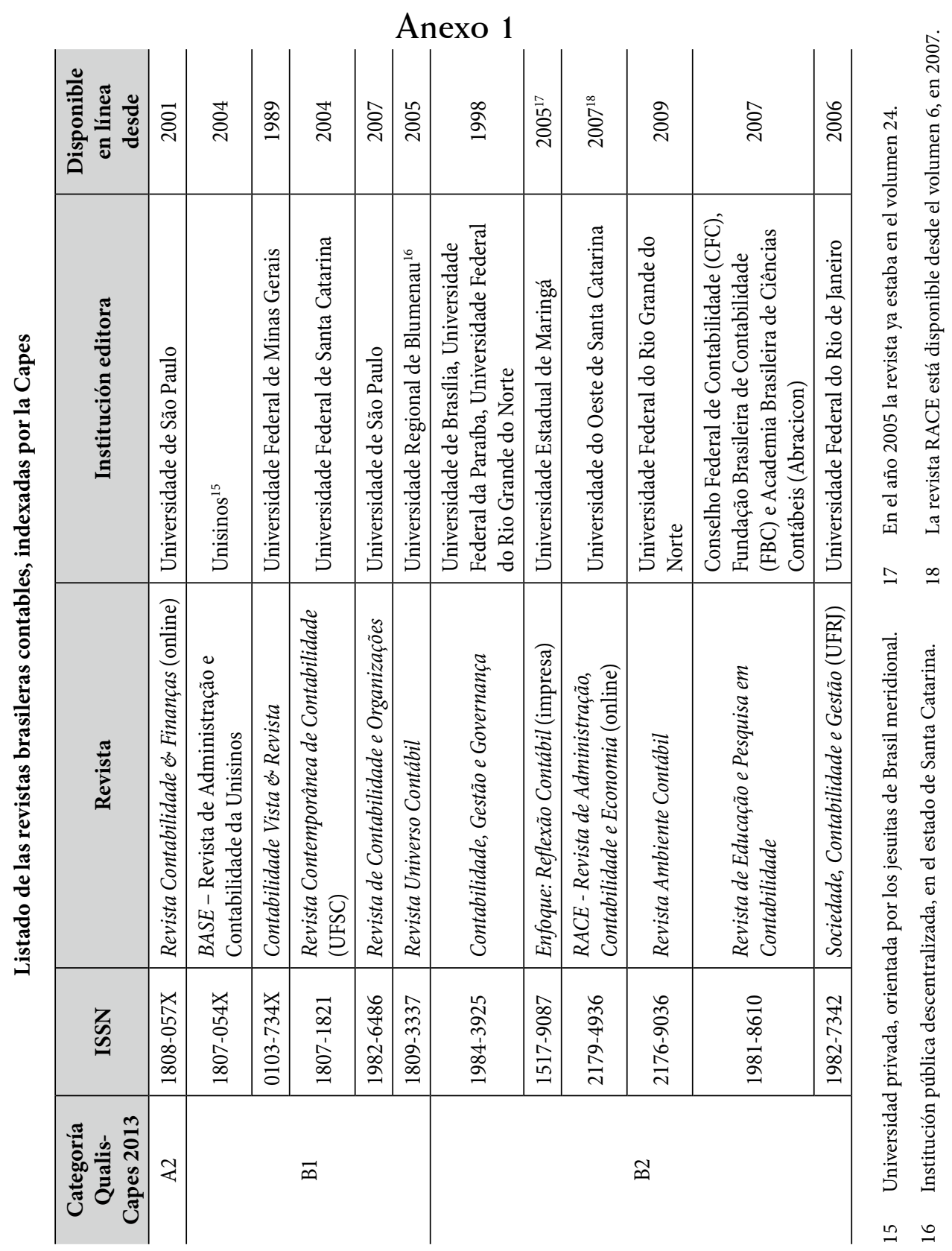




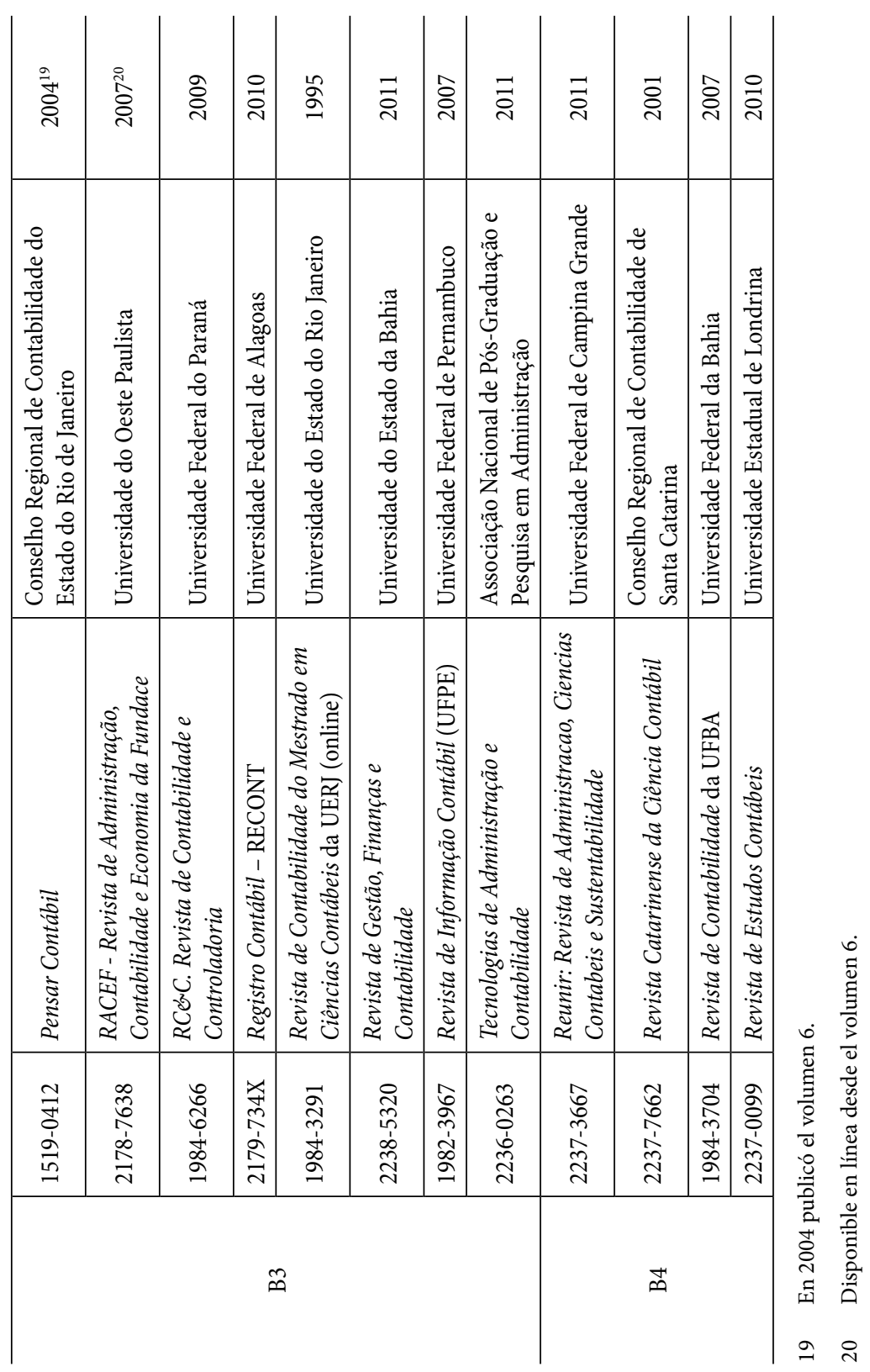




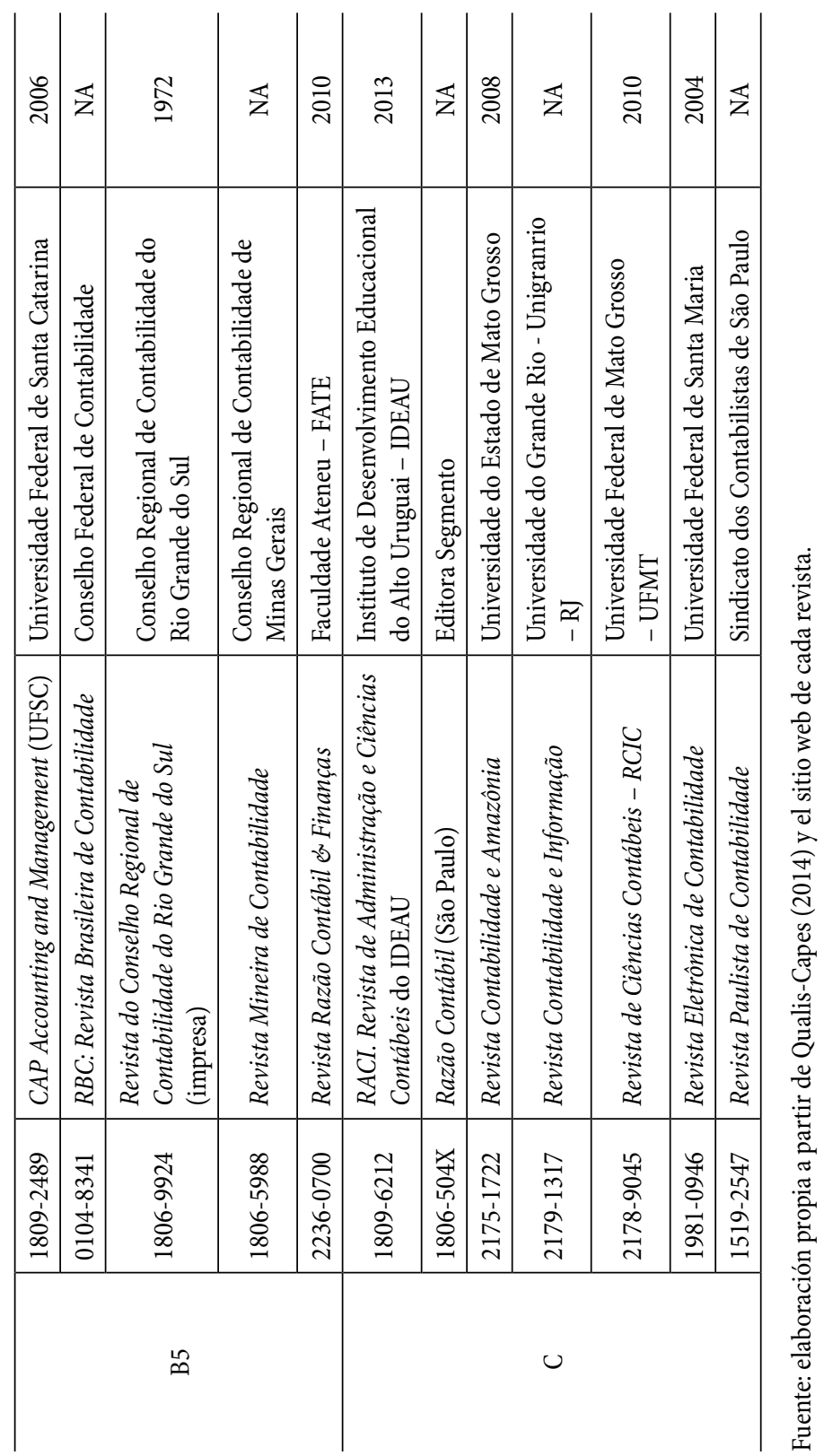

